\title{
A fast method for direction-of-arrival estimation
}

\author{
G SHARMA and A K RAINA \\ Department of Electrical Engineering, Indian Institute of Technology, Kanpur \\ 208016, India \\ e-mail: [govind, akraina]@iitk.ac.in
}

MS received 10 February 1998; revised 16 June 1999

\begin{abstract}
In many eigenvalue-eigenvector-based direction-of-arrival estimation algorithms, one finds orthogonal basis vectors for the noise subspace. We propose a circulant basis for the noise space which can be calculated more efficiently than an orthogonal basis. The new method for high signal-to-noise ratio (SNR) has a performance similar to the well-known MUSIC algorithm but can be implemented much more efficiently.
\end{abstract}

Keywords. Direction of arrival; signal and noise subspace-based method; circulant basis.

\section{Introduction}

The following matrix equation is encountered frequently in many eigenvalue-eigenvectorbased direction-of-arrival (DOA) estimation (Monzingo \& Miller 1980; Orfandis 1985; Pillai 1989; Jongtae \& Bose 1994):

$$
R=A R_{u} A^{H}+\sigma^{2} I,
$$

where $R$ is an $M \times M$ covariance matrix of the sensor outputs. $R_{u}$ is a $K \times K$ source covariance matrix for $K$ sources, $A$ is an $M \times K$ matrix consisting of $K$ direction vectors and $\sigma^{2}$ is noise variance of the sensor noise. Many algorithms like MUSIC etc. are based on the fact that $R$ can be decomposed into two orthogonal subspaces: (1) signal subspace of dimension $K$, and (2) a noise subspace of dimension $(M-K)$. Generally one finds an orthogonal basis for one or both the subspaces. This unitary decomposition becomes computationally expensive when the dimensions involved are large. Here we propose a circulant basis for the noise subspace which can be calculated more efficiently than an orthogonal basis. This method gives performance similar to the other methods but is computationally superior especially when the dimensions involved are large.

\section{A circulant basis for noise subspace}

Let $P$ be the right shift operator on $R^{M}$, so that $P$ in matrix form can be written as 


$$
P=\left(\begin{array}{ccccc}
0 & 0 & \cdots & 0 & 0 \\
1 & 0 & \cdots & 0 & 0 \\
0 & 1 & \cdots & 0 & 0 \\
\vdots & \vdots & & \vdots & \vdots \\
0 & 0 & \cdots & 1 & 0
\end{array}\right)
$$

The elements of the $A$ matrix are given by

$$
a_{m, n}=e^{-j d_{m} \omega_{n}}, m=1,2, \ldots, M, n=1,2, \ldots, K,
$$

where $d_{i}=i-1$ is the normalized distance between sensors and $\omega_{i}=\pi \cos \theta_{i}$ contain the direction-of-arrival information. With these definitions we have the following theorem.

Theorem 1. Let $M \geq 2 K$ and

$$
A^{H}=\left[\mathbf{1} \vdots A_{0} \vdots B_{0}\right]
$$

with 1 denoting a $K \times 1$ vector of all ones, $A_{0}, K \times K$ and $B_{0}, K \times M-K-1$ matrices being partitions of the A matrix. Then the set

$$
\left\{\mathbf{v}, P \mathbf{v}, \ldots, P^{M-K-1} \mathbf{v}\right\}
$$

where

$$
\mathbf{v}=\left(\begin{array}{c}
1 \\
\cdots \\
-A_{0}^{-1} \mathbf{1} \\
\cdots \\
\mathbf{0}
\end{array}\right)
$$

and where 0 denotes a $(M-K-1) \times 1$ vector of zeros, forms a basis set for the noise subspace.

Proof. First we show that $A_{0}^{-1}$ exists. We have

$$
A_{0}=\left(\begin{array}{cccc}
x_{1} & x_{1}^{2} & \cdots & x_{1}^{K} \\
x_{2} & x_{2}^{2} & \cdots & x_{2}^{K} \\
\vdots & & & \\
x_{K} & x_{K}^{2} & \cdots & x_{K}^{K}
\end{array}\right)
$$

where $x_{i}=e^{j \omega_{i}}, i=1,2, \cdots, K$

$$
\operatorname{det}\left(A_{0}\right)=\prod_{i=1}^{K} x_{i} \prod_{1 \leq j<k \leq K}\left(x_{j}-x_{k}\right)
$$

which is nonzero, if $x_{i} \neq 0$, and $x_{j} \neq x_{k}$ for $j \neq k$. This condition is obviously met in our case. Now consider 


$$
\begin{aligned}
A^{H} \mathbf{v} & =\left[\mathbf{1} \vdots A_{0} \vdots B_{0}\right]\left[\begin{array}{c}
1 \\
-A_{0}^{-1} \mathbf{1} \\
\mathbf{0}
\end{array}\right] \\
& =\mathbf{1}-A_{0} A_{0}^{-1} \mathbf{1} \\
& =\mathbf{0}
\end{aligned}
$$

We observe that if

$$
X_{j}^{H} \mathbf{y}=0,
$$

where

$$
X_{j}^{H}=\left[x_{j}^{0} x_{j}^{1} \cdots x_{j}^{k}\right]
$$

then

$$
x_{j}^{L} X_{j}^{H} \mathbf{y}=\mathbf{0}
$$

This implies that

$$
A^{H} \mathbf{x}=\mathbf{0} .
$$

for

$$
\mathbf{x} \in\left\{\mathbf{v}, P \mathbf{v}, \ldots, P^{M \cdot-K^{\prime-1}} \mathbf{v}\right\}
$$

Remark. The vectors $\mathbf{v}, P \mathbf{v}, \ldots, P^{M-K-1} \mathbf{v}$ are not orthogonal, but they form a basis set for the noise subspace.

We now show how to calculate these from the data. Let

$$
R_{0}=R-\sigma^{2} I
$$

then

$$
\begin{aligned}
R_{0} \mathbf{v} & =\left(R-\sigma^{2} I\right) \mathbf{v} \\
& =A R_{u} A^{h t} \mathbf{v} \\
& =\mathbf{0} .
\end{aligned}
$$

Let us partition $R_{\mathbf{t}}$ as follows:

$$
R_{0}=\left[\begin{array}{ccc}
R_{1} & \vdots & R_{3} \\
\cdots & \ldots & \cdots \\
R_{2} & \vdots & R_{4}
\end{array}\right]
$$

where $R_{1}$ is $(K+1) \times(K+1)$ and other $R_{i}$ s are of compatible dimensions. Now we have

$$
R_{0} \mathbf{v}=\mathbf{0}
$$




$$
=\left[\begin{array}{ccc}
R_{1} & \vdots & R_{3} \\
\cdots & \ldots & \ldots \\
R_{2} & \vdots & R_{4}
\end{array}\right]\left[\begin{array}{c}
1 \\
v_{1} \\
\vdots \\
v_{K} \\
0 \\
\vdots \\
0
\end{array}\right],
$$

so

$$
\left[\begin{array}{l}
R_{1} \\
R_{2}
\end{array}\right]\left[\begin{array}{c}
1 \\
v_{1} \\
\vdots \\
v_{K}
\end{array}\right]=\mathbf{0}
$$

Hence

$$
\operatorname{rank}\left[\begin{array}{c}
R_{1} \\
R_{2}
\end{array}\right] \leq K .
$$

Let $\mathbf{r}$ be the first column of

$$
\left[\begin{array}{l}
R_{1} \\
R_{2}
\end{array}\right] \text {. }
$$

Using the partition

$$
[\mathbf{r}: \bar{R}]
$$

of this, we get

$$
[\mathbf{r}: \bar{R}]\left[\begin{array}{c}
1 \\
v_{1} \\
\vdots \\
v_{K}
\end{array}\right]=\mathbf{0}
$$

or

$$
\bar{R}\left[\begin{array}{c}
v_{1} \\
v_{2} \\
\vdots \\
v_{K}
\end{array}\right]=-\mathbf{r}
$$

We note that rank $\vec{R} \leq K$. The equality always holds if $M \leq 2 K-1$. If $\bar{R}$ has full rank then we can solve the above equation directly using left inverse of $\bar{R}$. In cases where the rank of $R_{0}$ is not equal to $K$ (it is actually greater than $K$ in practical cases when $R$ is estimated from data) we use partial pivoting before solving the above equation. 


\section{Simulation results}

We have compared our method with the MUSIC algorithm. Dimensions of the signal subspace are estimated by comparing the eigenvalues of the sensor output correlation matrix and using a threshold. In all practical cases, the smallest eigenvalues (corresponding to the noise subspace) are not the same. Let

$$
\sigma_{S_{1}} \leq \sigma_{S_{2}} \leq \cdots \leq \sigma_{S_{K}} \leq \sigma_{N_{1}} \leq \cdots \leq \sigma_{N_{M K}}
$$

be the eigenvalues of the correlation matrix $R$, then last $(M-K)$ eigenvalues corresponding to the noise subspace are not equal. Hence the rank of $R_{0}=R-\sigma_{N_{1}} I$ is no longer $K$ but is greater than $K$.

The direction of arrival is estimated by locating the peaks of the spectrum given by

$$
P(\omega)=1 / \sum_{j=1}^{M-K}\left|a^{H}(\omega) V_{j}\right|^{2} .
$$

Simulations were carried out for $K=2$ and $M=3,4$ and 5. The DOAs were $30^{\circ}$ and $60^{\circ}$. The signal-to-noise ratio (SNR) was $20 \mathrm{~dB}$. We carried out 100 independent trials for the simulation and the results are as follows:

For $M=3, K=2$ there is no rank degeneracy in the new method and the following results were obtained.

$$
\begin{aligned}
& \text { MUSIC: Mean }=30.1^{\circ}, \text { var }=0.573 \text { and mean }=60^{\circ}, \text { var }=0.21 . \\
& \text { New method: } \quad \text { Mean }=30^{\circ}, \text { var }=0.51 \text { and mean }=60^{\circ}, \text { var }=0.17 .
\end{aligned}
$$

For $M=4, K=2$ there is rank degeneracy; thus we have done partial pivoting before determining the noise subspace basis vectors. We first searched for the largest element in the first column and then, by interchanging rows, put that element in the $(1,1)$ position. Similarly, the second column was searched for the largest element in magnitude and then that element brought to the $(2,2)$ position, and so on for the other columns. The last $M-K$ rows of $\vec{R}$ were forced to zero.

$$
\begin{aligned}
& \text { MUSIC: Mean }=30^{\circ}, \mathrm{var}=0.32 \text { and mean }=60^{\circ}, \mathrm{var}=0.17 . \\
& \text { New method: } \text { Mean }=30.2^{\circ} . \text { var }=0.645 \text { and mean }=60^{\circ}, \text { var }=0.215 .
\end{aligned}
$$

Similar results were obtained for $M=5, K=2$ case.

The performances of the two methods are quite similar at high SNRs (greater than or equal to $20 \mathrm{~dB}$ ). In the new method, we calculate only one eigenvector and find other basis vectors by shifting elements of this vector. If this estimate is noisy, then the error is carried over to all the basis vectors. Therefore at low SNRs. its performance is not as good as that of root-MUSIC.

\section{Computational requirements}

The MUSIC algorithm and the new method are quite similar. The steps involved are: (1) Determining the eigenvalues of the correlation matrix $R,(2)$ determining the basis vectors for the noise subspace, and (3) calculating the spectrum. The second step is different in the two methods. The MUSIC algorithm finds an orthogonal basis, while the new method finds 
a circulant basis. Determining eigenvalues is an iterative procedure and requires approximately $\frac{2}{3} M^{3}$ multiplications and $0.5 M^{2}$ square-root operations (Wilkinson 1965). Step 2 in MUSIC algorithm requires approximately $2.5(M-K) M^{2}$ multiplications, while this step in the new method requires approximately $2.5 M^{2}$ multiplications (Wilkinson 1965). Step 3 is the same in both the methods. Thus we see that the new method has significant computational advantage when the noise subspace dimension $(M-K)$ is large.

The two methods were compared on the basis of computational time. For $K=2$ and $M=5,7$ and 10, MUSIC method took (on the average) 13.3, 24 and 30 milliseconds respectively, whereas the new method took $6.66,6.8$ and 7.5 milliseconds respectively (based on average of 100 trials).

\section{Conclusions}

Simulation results show that the performance of the MUSIC and the new method are quite similar at high SNRs (greater than or equal to $20 \mathrm{~dB}$ ), while at very low SNRs root-MUSIC performs better than the proposed method. This is expected as both methods are using orthogonality of the signal and noise subspace. The advantage of the new method is in computational efficiency. As the number of sensors increases, the dimensions of the data correlation matrix increase. In our method one has to calculate only one basis vector, while the other vectors are obtained by shifting elements of this. The result is faster computation of the noise subspace basis. At low SNRs this becomes a disadvantage in terms of accuracy as we are calculating only one basis vector from the data and, if this estimate is noisy, then all the basis vectors have this noise.

\section{References}

Jongtae C, Bose N K 1994 A novel subspace based approach to parameter estimation. J. Digital Signal Process. 4: 40-48

Monzingo A, Miller W 1980 Introduction to adaptive arrays (New York: John Wiley and Sons)

Orfandis J 1985 Optimum signal processing: An introduction (New York: McMillan)

Pillai S U 1989 Array signal processing (New York: Springer Verlag)

Wilkinson J H 1965 The algebraic eigenvalue problem (Oxford: Clarendon) 